\title{
Loss of $A \beta$-nerve endings associated with the Merkel cell-neurite complex in the lesional oral mucosa epithelium of lichen planus and hyperkeratosis
}

\author{
Daniela Calderón Carrión ${ }^{1}$, Yüksel Korkmaz ${ }^{1,2,3}$, Britta $\mathrm{Cho}^{2}$, Marion Kopp ${ }^{1}$, Wilhelm Bloch ${ }^{4}$, Klaus Addicks ${ }^{3}$ \\ and Wilhelm Niedermeier ${ }^{1}$
}

The Merkel cell-neurite complex initiates the perception of touch and mediates A $\beta$ slowly adapting type I responses. Lichen planus is a chronic inflammatory autoimmune disease with T-cell-mediated inflammation, whereas hyperkeratosis is characterized with or without epithelial dysplasia in the oral mucosa. To determine the effects of lichen planus and hyperkeratosis on the Merkel cell-neurite complex, healthy oral mucosal epithelium and lesional oral mucosal epithelium of lichen planus and hyperkeratosis patients were stained by immunohistochemistry (the avidin-biotin-peroxidase complex and double immunofluorescence methods) using pan cytokeratin, cytokeratin 20 (K20, a Merkel cell marker), and neurofilament 200 (NF200, a myelinated A $\beta$ - and A $\delta$-nerve fibre marker) antibodies. NF200-immunoreactive (ir) nerve fibres in healthy tissues and in the lesional oral mucosa epithelium of lichen planus and hyperkeratosis were counted and statistically analysed. In the healthy oral mucosa, K20-positive Merkel cells with and without close association to the intraepithelial NF200-ir nerve fibres were detected. In the lesional oral mucosa of lichen planus and hyperkeratosis patients, extremely rare NF200-ir nerve fibres were detected only in the lamina propria. Compared with healthy tissues, lichen planus and hyperkeratosis tissues had significantly decreased numbers of NF200-ir nerve fibres in the oral mucosal epithelium. Lichen planus and hyperkeratosis were associated with the absence of $A \beta$-nerve endings in the oral mucosal epithelium. Thus, we conclude that mechanosensation mediated by the Merkel cell-neurite complex in the oral mucosal epithelium is impaired in lichen planus and hyperkeratosis. International Journal of Oral Science (2016) 8, 32-38; doi:10.1038/ijos.2015.31; published online 23 October 2015

Keywords: A $\beta$-nerve fibres; hyperkeratosis; lichen planus; mechanosensation; Merkel cell-neurite complex

\section{INTRODUCTION}

The receptors of the oral mucosa are classified as thermoreceptors, mechanoreceptors and nociceptors. Thermoreceptors are nerve endings that perceive warming and cooling stimuli. ${ }^{1}$ Mechanoreceptors are specialized receptors that perceive stretch, touch and pressure stimuli, ${ }^{1}$ while nociceptors are receptors that perceive painful stimuli. ${ }^{1-2}$ Compared with free nerve endings in the oral mucosa, the encapsulated nerve endings such as Pacinian corpuscles, Meissner corpuscles and Merkel cells are specialized mechanoreceptors.

The afferent nerve endings innervate the mucosa and skin are categorized by their conduction velocity into three groups: $\mathrm{A} \beta, \mathrm{A} \delta$ and $\mathrm{C}$ nerve fibres and nerve endings. ${ }^{1,3}$ The sensation of touch is mediated by $\mathrm{A} \beta$ fibres. Nociception is mediated by $\mathrm{A} \delta$ - and C-fibres. ${ }^{1}$ The $\mathrm{A} \beta$ nerve fibres are subdivided into three groups: slowly adapting type I and type II nerve fibres and rapidly adapting nerve fibres. The slowly adapting type I nerve fibres innervate Merkel cell-neurite complexes, ${ }^{1,4-5}$ while the slowly adapting type II nerve fibres innervate Ruffini corpuscles. ${ }^{1}$ The rapidly adapting nerve fibres innervate
Meissner and Pacinian corpuscles. ${ }^{1}$ Merkel cells have been proposed to be the mechanosensory receptor cells within the Merkel cell-neurite complexes because Merkel cells form synaptic contacts with somatosensory afferent nerve endings. ${ }^{1,4}$ The Merkel cell-neurite complex is a unique sensory unit that mediates mechanosensation. ${ }^{6-7}$ Merkel cells respond to touch stimuli and induce an action potential on the afferent nerve fibres that innervate the cells. ${ }^{6-7}$

The cutaneous peripheral nervous system is known to be involved in epidermal homeostasis. For example, the proliferation of keratinocytes is modulated by skin innervation. ${ }^{8}$ Sensory neuropeptide substance $\mathrm{P}$ regulates skin immunity via its receptor, NK-R $1,{ }^{9}$ while the neuropeptide calcitonin gene-related peptide (CGRP) is involved in the regulation of local keratinocyte growth. ${ }^{10}$ In the epidermis, the neuropeptide CGRP may have inhibitory immunomodulatory effects on Langerhans cell-mediated antigen presentation. ${ }^{11-12}$

It was described that reduced sensory perception by the oral mucosa epithelium following loss of teeth is compensated by an increase in the local Merkel cell numbers of the oral mucosa epithelium. ${ }^{13}$ From these

${ }^{1}$ Department of Prosthetic Dentistry, Dental School, University of Cologne, Cologne, Germany; ${ }^{2}$ Center for Biochemistry, Institute for Experimental Dental Research and Oral

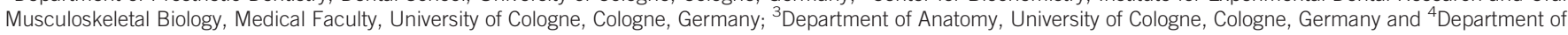
Molecular and Cellular Sport Medicine, German Sport University, Cologne, Germany


32, Cologne 50931, Germany

E-mail: yueksel.korkmaz@uk-koeln.de

Accepted 15 July 2015 
results, it was concluded that the reduction in the perception by the oral mucosal epithelium is compensated by an increase in the local Merkel cell population. ${ }^{13}$ These results indicate that Merkel cells are involved in the regulation of mechanosensation in the healthy oral mucosal epithelium.

In the oral mucosa, lichen planus is a chronic inflammatory autoimmune disease that involves T-cell-mediated inflammation. Oral hyperkeratosis is characterized by epithelial hyperkeratosis and hyperparakeratosis, with or without epithelial dysplasia. Under the effects of several different harmful irritants that trigger lichen planus and hyperkeratosis, cells of the oral mucosal epithelium reveal different changes associated with inflammation, degeneration, hyperplasia or dysplasia. To avoid non-physiological harmful stretch and pressure stimuli in the design of dental prosthodontics treatment, knowledge regarding mechanosensation in oral mucosal diseases, including lichen planus and hyperkeratosis, is of major importance. However, the effects of lichen planus and hyperkeratosis on the relationship between Merkel cells and nerve endings in the oral mucosa are not known.

The finding that cytokeratin 20 (K20) is a reliable marker for cutaneous Merkel cells ${ }^{14}$ was also confirmed in Merkel cells of the oral mucosa. ${ }^{15}$ To determine the effects of lichen planus and hyperkeratosis on the Merkel cell-A $\beta$-nerve ending complex in the oral mucosal epithelium, the localization and colocalization of neurofilament 200 (NF200, a marker for A $\beta$-nerve endings) and K20 were investigated by immunohistochemistry using an avidin-biotin-peroxidase complex and confocal double immunofluorescence methods in the healthy human oral mucosa epithelium and in lesional oral mucosa epithelium biopsies of lichen planus and hyperkeratosis patients.

\section{MATERIALS AND METHODS}

\section{Tissue sample collection}

Samples with lichen planus $(n=8)$ and hyperkeratosis $(n=6)$ originated from the buccal oral mucosa, while normal samples originated from the oral mucosa of the retromolar $(n=2)$ and palatal $(n=2)$ regions. Merkel cell numbers in the oral mucosa vary depending on the site (i.e., keratinized or non-keratinized oral mucosa).

Healthy oral mucosa samples from patients who were treated by plastic surgery were collected. The patients agreed to have their tissues examined for research purposes. The procurement of human oral mucosa samples at the time of surgery was approved by the Human Ethics Committee of Heinrich-Heine-University, Düsseldorf.

\section{The clinical and pathological characterization of tissue samples} Clinical histories were collected, and investigations of the subjects were performed to diagnose lichen planus and hyperkeratosis in the Clinic and Polyclinic for Oral and Maxillofacial Surgery of the University of Cologne. The histopathological diagnosis was confirmed by hematoxylin and eosin (H\&E) staining.

\section{Tissue fixation, embedding and sectioning}

The tissue samples were prepared and collected in a fixative containing $4 \%$ paraformaldehyde and $0.2 \%$ picric acid solution in $0.1 \mathrm{~mol} \cdot \mathrm{L}^{-1}$ phosphate-buffered saline (PBS; $\mathrm{pH}$ 7.4). The tissue samples were washed in $0.1 \mathrm{~mol} \cdot \mathrm{L}^{-1} \mathrm{PBS}\left(\mathrm{pH} 7.4\right.$ ) at $4{ }^{\circ} \mathrm{C}$ and embedded in paraffin. Using a microtome (HM 355 S Microtom; Microm International $\mathrm{GmbH}$, Walldorf, Germany), the samples were cut into $10-\mu \mathrm{m}$-thick sections.

\section{Hematoxylin and eosin staining}

The sections were deparaffinized in xylenes and rehydrated in a series of ethanol washes at decreasing concentrations. Then, the sections were washed in distilled water and immersed into Mayer's hematoxylin solution for $10 \mathrm{~min}$, washed in running tap water, rinsed in distilled water for $2 \mathrm{~min}$ and then immersed in eosin solution for $1 \mathrm{~min}$. The sections were dehydrated in ethanol, cleared in xylenes and mounted in Entellan (Merck, Darmstadt, Germany).

\section{Immunohistochemistry}

Avidin-biotin-peroxidase complex method. The deparaffinized sections were washed in $0.05 \mathrm{~mol} \cdot \mathrm{L}^{-1}$ tris(hydroxymethyl)aminomethane (Tris)-buffered saline (TBS), and the sections were treated with proteinase $\mathrm{K}$ (DAKO, Hamburg, Germany) for $3 \mathrm{~min}$. The endogenous peroxidase activity was inhibited by $0.05 \mathrm{~mol} \cdot \mathrm{L}^{-1} \mathrm{TBS}$ containing $0.3 \% \mathrm{H}_{2} \mathrm{O}_{2}$. Then, the sections were treated with $0.25 \%$ Triton $\mathrm{X}-100$. The non-specific-binding sites for antibodies were blocked with $2 \%$ bovine serum albumin (BSA; PAA Laboratories $\mathrm{GmbH}$, Cölbe, Germany) and 5\% normal goat serum (NGS; Vector Laboratories, Burlingame, CA, USA). Subsequently, the sections were incubated with the following antibodies: mouse monoclonal anti-pan cytokeratin (pK, 1:100; Sigma, St. Louis, MO, USA), rabbit anti-K20 (1:250, Abcam, Cambridge, UK) and mouse anti-NF200 (1:2 000, Sigma, Taufkirchen, Germany). The sections were incubated with biotinylated goat anti-mouse (1:50) and goat anti-rabbit $\operatorname{IgG}(1: 100$, Vector Laboratories, Burlingame, CA, USA), as appropriate, corresponding to the primary antibodies. Then, the sections were incubated in avidin-biotin-peroxidase complex (1:100; Vector Laboratories, Burlingame, CA, USA) and the immunohistochemical-binding sites were visualized with $0.05 \%$ 3,3' -diaminobenzidine tetrahydrochloride (Sigma, Taufkirchen, Germany) in $0.05 \mathrm{~mol} \cdot \mathrm{L}^{-1}$ Tris-HCl buffer, $\mathrm{pH}$ 7.6, containing $0.01 \% \mathrm{H}_{2} \mathrm{O}_{2}$ and $0.01 \%$ nickel ammonium sulphate. The sections were mounted in Entellan (Merck, Darmstadt, Germany). Incubations without primary antibodies but with secondary antibodies (affinity-purified biotinylated goat anti-mouse IgG (Vector Laboratories, Burlingame, CA, USA) and affinity-purified biotinylated goat anti-rabbit IgG (Vector Laboratories, Burlingame, CA, USA) were carried out as controls, as previously described. ${ }^{16}$

Double immunofluorescence. The sections were deparaffinized and treated with proteinase K (DAKO, Hamburg, Germany) for 3 min. The non-specific-binding sites for the antibodies were blocked with blocking solution containing 2\% BSA and 5\% NGS. The sections were incubated with mouse monoclonal anti-NF200 (1:2 000; Sigma, Taufkirchen, Germany) antibody overnight at $4{ }^{\circ} \mathrm{C}$. Then, the sections were incubated with 488-DyLight-conjugated goat anti-mouse IgG (Pierce Biotechnology, Rockford, IL, USA) at 1:1 000. After blocking of the non-specific-binding sites by using a blocking solution containing $2 \%$ BSA and $5 \%$ NGS, the sections were incubated with rabbit polyclonal anti-cytokeratin 20 (K20) (Abcam, Cambridge, UK) at a 1:250 dilution in $0.05 \mathrm{~mol} \cdot \mathrm{L}^{-1} \mathrm{TBS}$ overnight at $4{ }^{\circ} \mathrm{C}$. Then, the sections were incubated with 550-DyeLight conjugated goat anti-rabbit IgG (Pierce Biotechnology, Rockford, IL, USA) at a dilution of 1:500. For cell nucleus identification, the sections were incubated with DRAQ5 (Axxora, Lörrach, Germany). The sections were mounted in Aqua Poly/Mount (Polysciences, Warrington, PA, USA). In double immunohistochemical incubations, controls were performed by incubating sections without the first (NF200) and second (K20) primary antibodies but with DRAQ5 to stain DNA and with secondary antibodies (488-DyLight-conjugated goat anti-mouse IgG and 550-DyLight-conjugated anti-rabbit IgG), as previously described. ${ }^{17}$ 


\section{Confocal image analysis}

Three colour fluorescent images were acquired on an LSM 510 META confocal microscope (Carl Zeiss, Oberkochen, Germany). The 488-nm excitation beam of an argon-krypton laser and a 505$530-\mathrm{nm}$ band-pass emission filter were used to selectively view the green fluorochrome (for the identification of NF200). The 543-nm excitation beam and 560-613-nm band-pass emission filter were used to selectively view the red fluorochrome (for the identification of K20). The $633 \mathrm{~nm}$ excitation beam and 649-702-nm band-pass emission filter were used to selectively view the far-red fluorochrome (for the identification of DRAQ5). ${ }^{17}$

\section{Quantification of nerve fibres and statistical analysis}

In the immunohistochemical incubations, consecutive sections of the oral mucosa were stained with pK, K20 and NF200 using the avidinbiotin complex method. The nerve fibres and nerve endings were identified for quantification by staining for NF200. For the quantification, one section per patient was selected. The quantification was performed in four sections $(n=4)$ from four healthy individuals, in eight diseased lichen planus sections $(n=8)$ from eight patients and in six diseased hyperkeratosis sections $(n=6)$ from six patients.

NF200-immunoreactive (ir) nerve fibre profiles were visualized using conventional light microscopy. NF200-ir sections were viewed at $\times 10$ magnification using an Olympus microscope (Olympus Deutschland GmbH, Hamburg, Germany) attached to a phosphatidylcholines containing Cell F imaging software (Olympus Soft Imaging Solutions GmbH, Münster, Germany) Images were coded to blind the investigator to the patient number and status.

Nerve fibres and nerve endings distributed in the lamina propria, in the basal cell layer and in the intraepithelial area were fixed in a $10^{6} \mu \mathrm{m}^{2}$ area. Using a computerized image analysis system (Cell F ; Olympus Soft Imaging Solutions GmbH, Münster, Germany), the branching of nerve fibre bundles (as two or three units, depending on the branching of the nerve fibre bundles), of each nerve fibre (as one unit), across sectioned nerve fibres (as one unit) and of exclusive free nerve endings (as one unit) were counted per sections. Then, the numbers of the nerve fibres in the lesional oral mucosa of lichen planus and hyperkeratosis patients were statistically analysed.

Data were presented as the mean \pm standard error of the mean (SEM). The results were analysed using one-way analysis of variance, and the differences between the healthy and the diseased with lichen planus and hyperkeratosis groups were compared using Bonferroni's post hoc test. Statistical significance was defined as $P<0.05$.

\section{RESULTS}

In the healthy oral mucosa, epithelial cells formed an intact epithelial barrier with regular rete pegs. In the lamina propria, collagen fibres, blood vessels, nerve fibres, and stromal cells showed a regular cellular architecture (data not shown). In lichen planus, the epithelium was thin, and numerous inflammatory cells were identified in the basal layer as well as in the lamina propria. Regarding hyperkeratosis, sections from four patients revealed non-dysplastic changes, and sections from two patients revealed dysplastic changes.

pK expression in keratinocytes and Merkel cells, K20 expression in Merkel cells and NF200 expression in the nerve fibres and nerve endings of the oral mucosa

Compared with the moderate staining of keratinocytes, strong staining intensity for $\mathrm{pK}$ was detected in Merkel cells lying at the basal layer of the oral mucosa (Figure 1a). Strong K20 (a specific Merkel

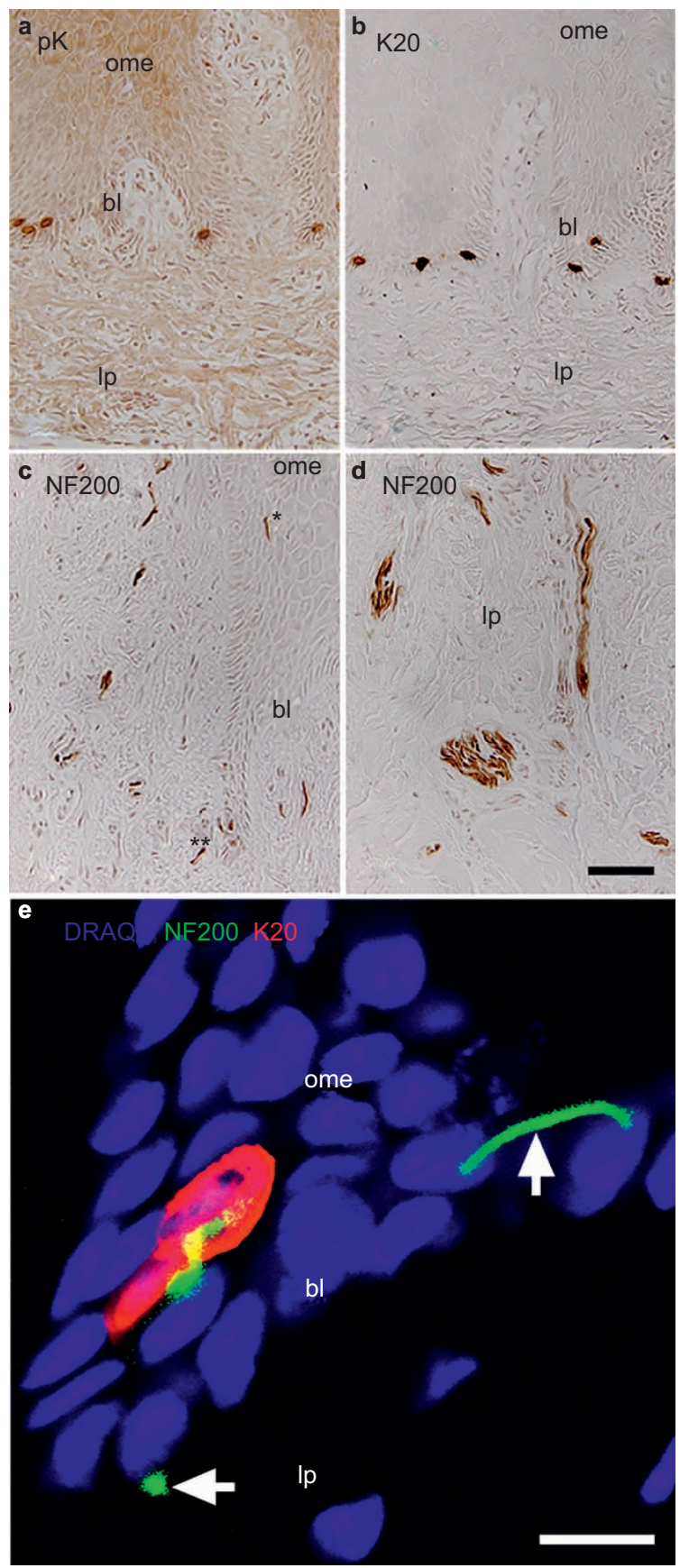

Figure 1 The expression of pK in keratinocytes and K20 in Merkel cells and the localization of NF200 in nerve fibres of healthy human oral mucosa. (a) In the healthy oral mucosal epithelium, pK is detected with different staining intensities (dependent on the epithelial layer) in keratinocytes and in Merkel cells of the oral mucosal epithelium. (b) K20 is identified only in Merkel cells located at the basal layer of the epithelium. (c and d) NF200 is located in nerve fibres and nerve endings distributed in the intraepithelial area (one asterisk), in the basal layer of the epithelium (two asterisks) and in the lamina propria of the healthy oral mucosa. (e) DRAQ5 (chromosome marker) is identified in cell nuclei of the keratinocytes (blue). Intraepithelial NF200-ir nerve fibres (green) are detected in association (yellow) with the K20-ir Merkel (red) cells at the basal layer of the epithelium. Note that some intraepithelial NF200-ir nerve fibres are not associated with the Merkel cells (arrows). Bars: $a-d=$ $50 \mu \mathrm{m} ; \mathrm{e}=10 \mu \mathrm{m}$. bl, basal layer; ir, immunoreactive; K20, cytokeratin 20; Ip, lamina propria; NF200, neurofilament 200; ome, oral mucosal epithelium; $\mathrm{pK}$, pan cytokeratin. 
cell marker)-staining intensity was identified only in the Merkel cells (Figure 1b). NF200-ir nerve fibres and nerve endings were detected in the epithelium, the basal layer (Figure 1c) and the lamina propria (Figure 1d) of the oral mucosa. By confocal double immunofluorescence staining, NF200-ir nerve fibres and nerve endings were detected; some of these nerve fibres and nerve endings were closely associated with the Merkel cells at the basal layer of the epithelium, whereas some detected intraepithelial NF200-ir nerve fibres and endings were not closely associated with the Merkel cells of the healthy oral mucosal epithelium (Figure 1e).
The loss of NF200-ir nerve fibres and nerve endings in the lesional oral mucosa epithelium of lichen planus

The lesional oral mucosa epithelium of lichen planus was characterized by $\mathrm{H} \& \mathrm{E}$ staining (Figure 2a and $2 \mathrm{~b}$ ). In lichen planus tissue sections, the epithelium was usually thin, (Figure 2a) and rete pegs were not detectable (Figure 2b). Numerous inflammatory cells were identified in the basal layer as well as in the lamina propria of the diseased oral mucosa (Figure 2b). In inflammation-associated cases, structural changes were observed in the basal layer and in the epithelium (Figure 2b). Keratinocytes of the oral mucosal epithelium and Merkel cells lying at

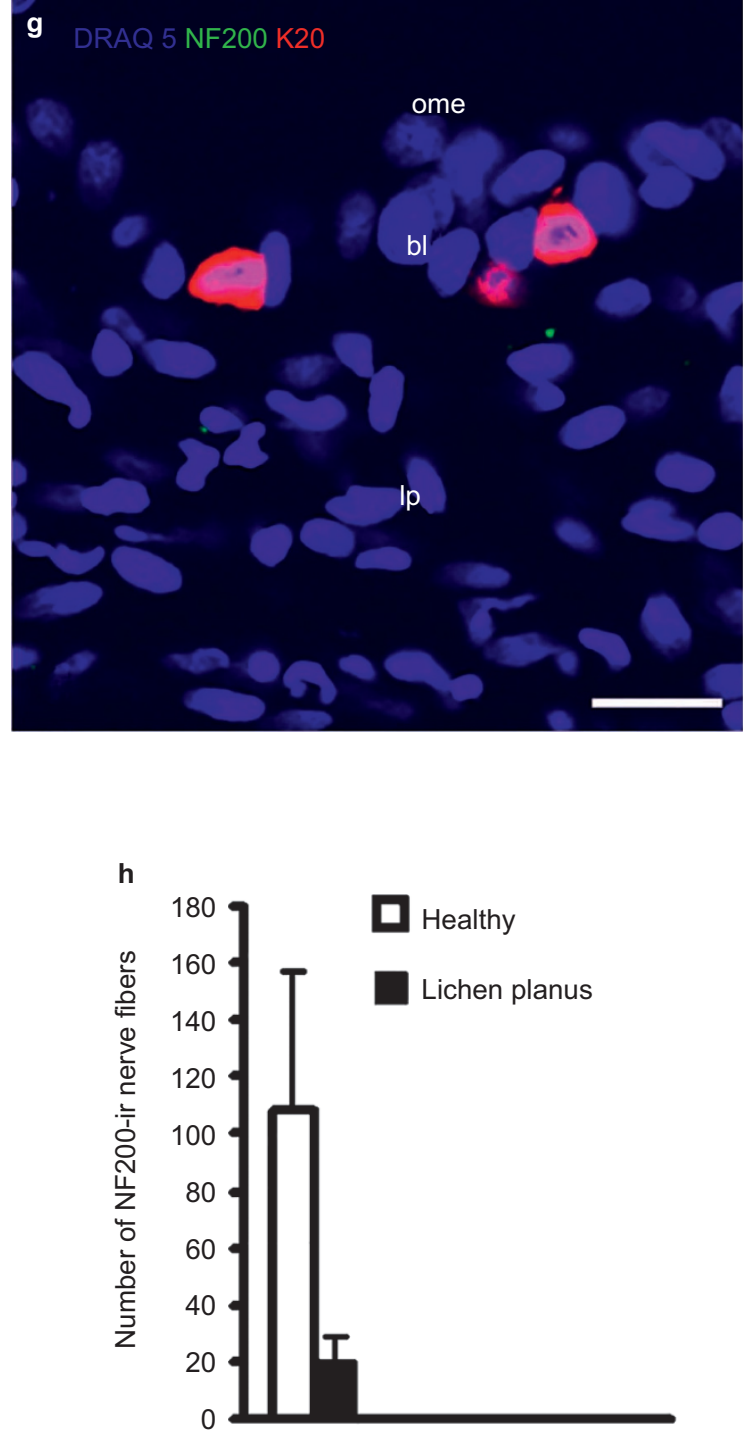

Figure 2 The expression of pK in keratinocytes and K20 in Merkel cells and the localization of NF200 in nerve fibres of the lesional human oral mucosa of lichen planus. (a) In lichen planus, the epithelium is thin, and rete pegs are not identified by H\&E staining. (b) The basal layer is not intact, and there are numerous lymphocytes with chronic inflammation in the lamina propria (detail of the squared area in a). (c) Keratinocytes are moderately positive for pK. Merkel cells are strongly positive for pK. (d) Only Merkel cells located at the basal layer are positive for K20. (e and f) Compared with the healthy oral mucosa, the lesional oral mucosa of lichen planus is associated with a significantly decreased number of NF200-ir nerve fibres distributed in the lamina propria (asterisks). (g) DRAQ5 is detected in the nuclei of cells of the diseased lichen planus tissue section. NF200-ir nerve fibres and nerve endings associated with the K20-ir Merkel cells are not detected. (h) The lesional oral mucosa of lichen planus is associated with a significantly decreased number of NF200-ir nerve fibres compared with the healthy oral mucosa. Bar: $\mathrm{a}=1 \mathrm{~mm} ; \mathrm{b}-\mathrm{f}=50$ $\mu \mathrm{m} ; \mathrm{g}=20 \mu \mathrm{m}$. bl, basal layer; H\&E, hematoxylin and eosin; ir, immunoreactive; K20, cytokeratin 20; Ip, lamina propria; NF200, neurofilament 200; ome, oral mucosal epithelium; pK, pan cytokeratin. 
the basal layer of the epithelium revealed positive staining for $\mathrm{pK}$ (Figure 2c). Strong K20-staining intensity was observed in Merkel cells of the lesional oral mucosa epithelium of lichen planus (Figure 2d).

NF200-ir nerve fibres and nerve endings were not detected in the lesional buccal mucosa epithelium of lichen planus (Figure 2e). In the lesional oral mucosa of lichen planus, NF200 was identified only in single nerve fibres and nerve endings distributed in the lamina propria (Figure 2e and 2f). K20-positive Merkel cells were identified without an association with nerve fibres and nerve endings (Figure $2 \mathrm{~g}$ ). Lichen planus was associated with a significant decrease in the number of NF200-positive nerve fibres found in the oral mucosa compared with the number found in the healthy oral mucosa (Figure $2 \mathrm{~h}$ ).

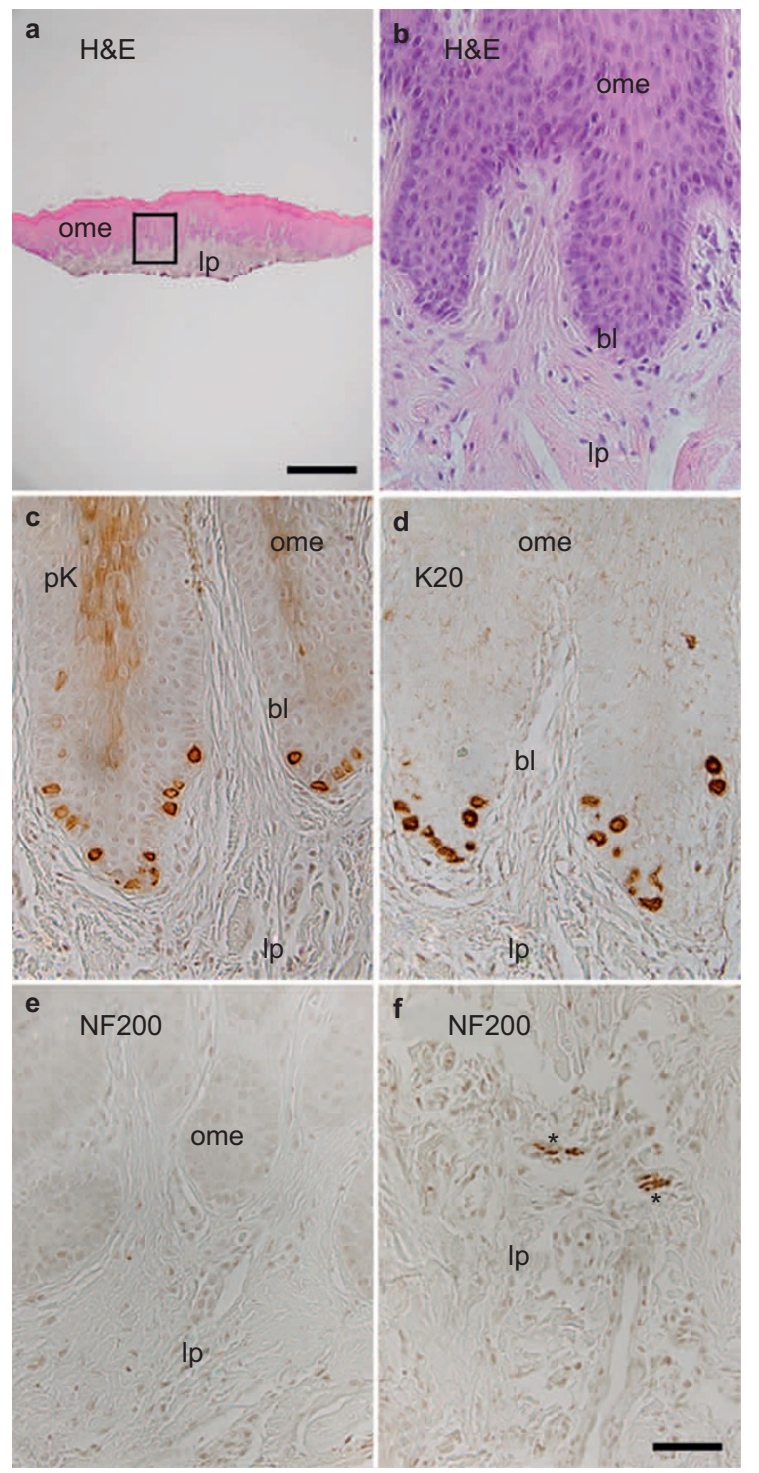

The loss of NF200-ir nerve fibres and nerve endings in the lesional oral mucosa epithelium of hyperkeratosis

The lesional oral mucosa epithelium of hyperkeratosis was characterized by conducting H\&E staining (Figure $3 \mathrm{a}$ and $3 \mathrm{~b}$ ). The cell nuclei of keratinocytes at the basal layer were larger than nuclei of the keratinocytes in the other oral mucosal epithelial layers (Figure 3b). Numerous large oval basal epithelial cell extensions into the underlying connective tissue were detected (Figure $3 \mathrm{~b}$ ). In some cases of hyperkeratosis, occasional inflammatory cells were identified (data not shown). In the lesional buccal epithelium of hyperkeratosis, $\mathrm{pK}$ was detected in the keratinocytes (Figure 3c). $\mathrm{K} 20$ was detected with strong staining intensity in the cytoplasm
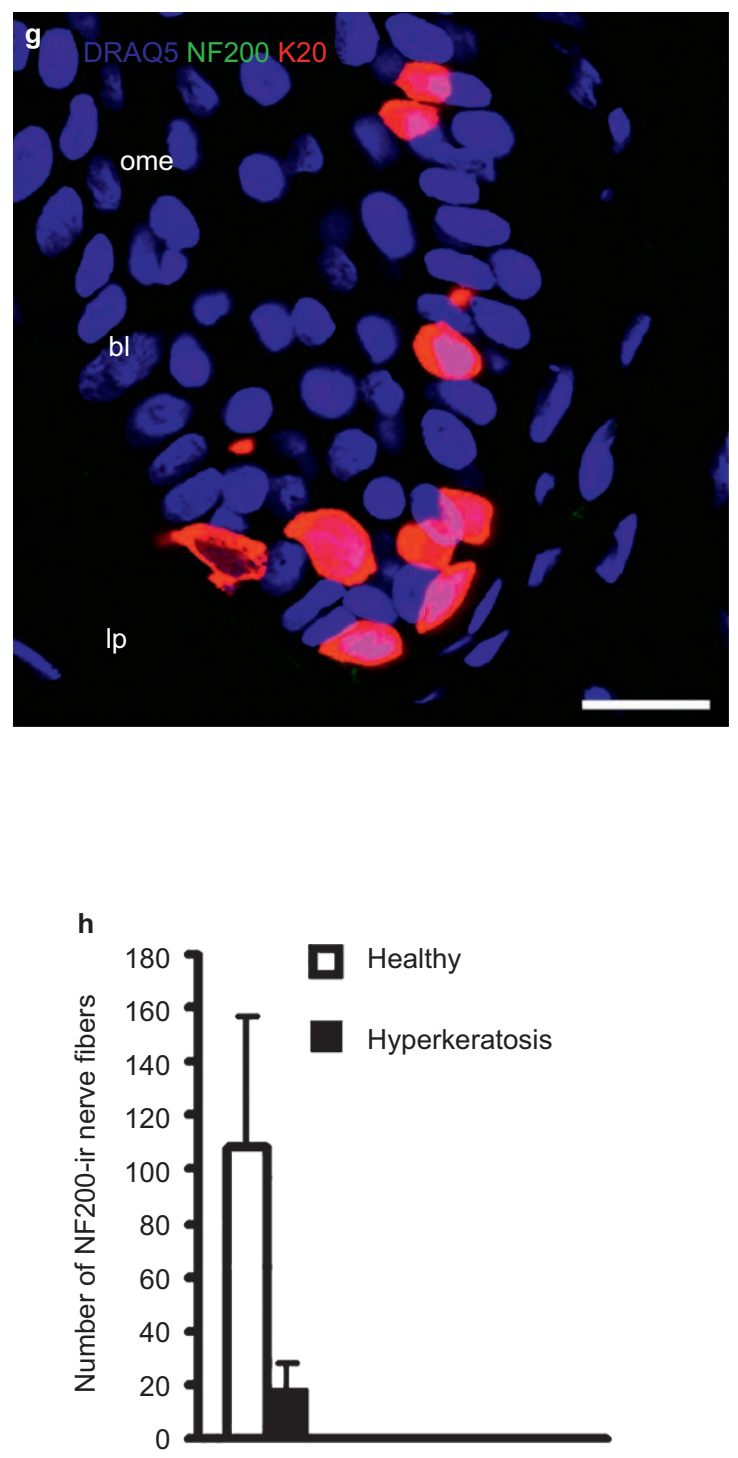

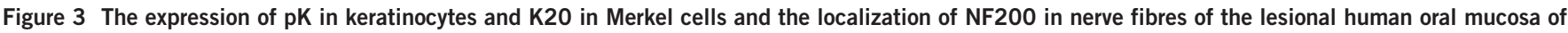

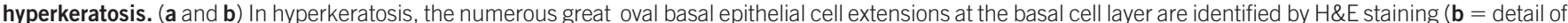

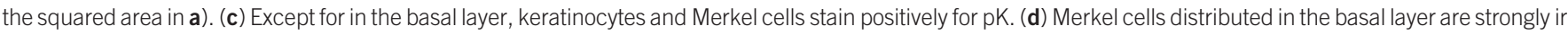

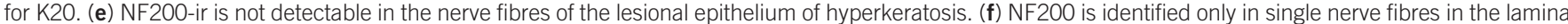

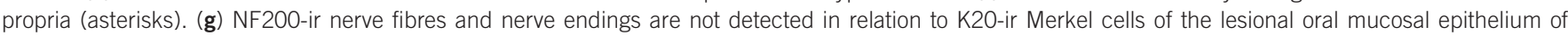

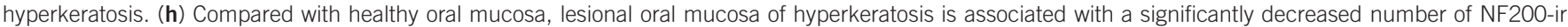

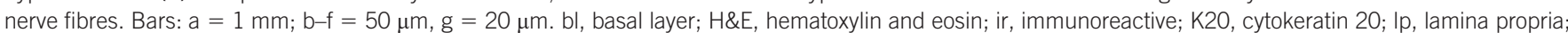
NF200, neurofilament 200; ome, oral mucosal epithelium; pK, pan cytokeratin. 
and with weak staining intensity in the nuclei of Merkel cells (Figure 3d).

In the lesional oral mucosal epithelium of hyperkeratosis, NF200 immunoreactivity was not detected in the intraepithelial area and at the basal layer of the epithelium (Figure 3e). Only single nerve fibres and nerve endings in the lamina propria were positive for NF200 (Figure 3f). K20-stained Merkel cells were detected without an association with nerve fibres or nerve endings in the lesional oral mucosal epithelium of hyperkeratosis (Figure $3 \mathrm{~g}$ ). Hyperkeratosis was associated with a significant decrease in the number of NF200-positive nerve fibres detected in the oral mucosa compared with the number identified in the healthy oral mucosa (Figure 3h).

\section{Immunohistochemical controls}

In control incubations, non-specific immunoreactivity was not detected (data not shown). In double confocal immunofluorescence staining, control incubations without the first and second primary antibodies resulted in disappearance of the specific immunohistochemical reaction (data not shown).

\section{Statistical analysis}

In the sections of healthy oral mucosa, numerous NF200-ir nerve fibres and nerve endings were counted in the lamina propria and intraepithelial area $(n=4 ;(108.86 \pm 47.90)$ densitometric units (DUs)). In tissues from patients with lichen planus and especially hyperkeratosis, nerve fibres were only detected rarely in the lamina propria. Compared with the healthy oral mucosa, the oral mucosa from lichen planus patients is associated with a significantly reduced number of NF200-ir nerve fibres $(n=8 ;(20.08 \pm 9.11)$ DUs $)$ (Figure 2h). Regarding hyperkeratosis ( $n=6$; $(17.77 \pm 10.25)$ DUs), the number of NF200-ir nerve fibres identified was significantly decreased compared with the NF200-ir nerve fibres and nerve endings identified in the healthy oral mucosa (Figure $3 \mathrm{~h}$ ).

\section{DISCUSSION}

In the healthy human oral mucosa, Merkel cells were detected in association with intraepithelial NF200-ir nerve fibres. Lichen planus and hyperkeratosis were associated with a loss of NF200-ir nerve fibres and nerve endings in the oral mucosal epithelium. The loss of the association between nerve endings and Merkel cells was similar in hyperkeratosis with non-dysplastic changes and with dysplastic changes. From these results, it may be suggested that the lichen planus-, and hyperkeratosis-associated loss of nerve endings in the Merkel cell-neurite complex of the buccal mucosa results in impairment of the Merkel cell-neurite complex-modulated perception of touch signalling in the oral mucosal epithelium.

In healthy buccal mucosal epithelium, NF200 (a marker of myelinated sensory $\mathrm{A} \beta$ - and $\mathrm{A} \delta$-nerve fibres) was detected in intraepithelial nerve endings in close association with keratinocytes. It was shown that keratinocyte proliferation at the epithelial basal layer was modulated by $A \beta$ - and $A \delta$-nerve fibres and nerve endings. ${ }^{8}$ In addition to the noxious stimulus transduction of external stimuli to the central nervous system, evidence suggests that sensory nerve fibres influence keratinocytes $^{9-10}$ and Langerhans cells ${ }^{11-12}$ by releasing sensory neuropeptides. It is known that sensory $A \beta$ - and $A \delta$-nerve fibres contain abundant sensory neuropeptides, especially substance P and CGRP. Therefore, it is possible that the function of the oral mucosal epithelium is modulated by sensory nerve fibres by a balance between the growth and differentiation of keratinocytes, which may be regulated by the sensory neuropeptides substance P and CGRP under physiological conditions. In contrast to the healthy buccal mucosal epithelium, the absence of the $\mathrm{A} \beta$ - and $\mathrm{A} \delta$-nerve fibres and nerve endings in diseased oral mucosal epithelium indicates that the neuropeptidedependent regulation of Langerhans cells and keratinocytes is impaired in the cases of lichen planus and hyperkeratosis. It is clear that keratinocytes may be affected by lichen planus and hyperkeratosis in a lesion-dependent manner by a number of other unknown complex mechanisms.

Merkel cells are required for touch sensitivity. ${ }^{118}$ In in vitro ${ }^{19}$ and in vivo ${ }^{1}$ experiments, it was found that a close relationship exists and that contact occurs between nerve endings and Merkel cells. Merkel cells contain dense-core vesicles that resemble neurosecretory vesicles. ${ }^{20}$ The reciprocal synapses were detected at the contact regions between Merkel cell plasma membrane and neurite plasma membrane. ${ }^{21}$ Merkel cells express the molecular tools required to send excitatory as well as modulatory signals to sensory neurons. ${ }^{4}$ In Merkel cells, the expression of neuronal transcriptional factors, voltage-gated ion channels (voltage-gated $\mathrm{K}^{+}$-channel subunits) and synaptic proteins was demonstrated. ${ }^{4}$ These results support the notion that Merkel cells are sensory receptor cells that transmit touch signals to the nerve endings of somatosensory neurons through synaptic contacts. Finally, it was shown that the depolarization of Merkel cells induced action potentials in slowly adapting type I afferent nerve fibres that innervated Merkel cells. ${ }^{6}$ In contrast, the hyperpolarization of Merkel cells suppressed touch-induced action potentials. ${ }^{6}$ In this mechanosensation process, Piezo22, a mechanical activated cation channel, is required for touch-induced activity in Merkel cells. ${ }^{7}$ These results indicate that Merkel cells regulate mechanosensation by inducing action potentials in the afferent nerve fibres in response to touch stimuli. In the present study, the close relationship between NF200-positive nerve endings and K20-positive Merkel cells of the healthy buccal mucosa indicated that physiological touch perception exists in the buccal mucosal epithelium. Merkel cells are mechanosensory cells that activate $\mathrm{A} \beta$-sensory afferent endings in the healthy buccal mucosa.

There is evidence ${ }^{22-23}$ supporting the finding that the Merkel cellneurite complex unit is required for intact mechanosensory touch signalling. The removal of Merkel cells by enzymatic treatment, photoablation or gene knockout resulted in absence of the responses of slowly adapting afferents. ${ }^{22-23}$ It is therefore possible that the lichen planus- and hyperkeratosis-associated loss of nerve endings from the Merkel cell-neurite complex unit results in the impairment of touch signalling perception in the buccal mucosal epithelium. Compared with lichen planus, hyperkeratosis was associated with a significant decrease in the number of the NF200-positive nerve fibres and nerve endings in the lamina propria. Lichen planus- and hyperkeratosisassociated loss of afferent myelinated $A \beta$-nerve fibres and nerve endings may be affected in a lesion-dependent manner by different mechanisms. Therefore, elucidating the definitive lesion-specific mechanisms responsible for lichen planus- and hyperkeratosisassociated loss of the relationship between Merkel cells and afferent myelinated $A \beta$-nerve endings requires additional experiments.

The extraction of teeth induces a reduction in the perception of the oral mucosal epithelium. This reduction is associated with an increase in the local Merkel cell population, indicating that the reduction in the perception of the oral mucosal epithelium may be compensated by an increase in the number of local Merkel cells. ${ }^{13}$ This is of great importance for the role of Merkel cells in the regulation of mechanosensation of the healthy oral mucosa. Because activation of the touch receptors is 
impaired in lichen planus and in hyperkeratosis, in the prosthetic treatment of teeth, the whole oral cavity, especially the buccal mucosa, must be clinically observed and examined. Therefore, the administration of a prosthetic therapy requires a healthy oral mucosal epithelium; healthy innervation and mechanosensation are prerequisites for protection of the oral mucosal epithelium against harmful stretch and pressure stimuli, which may be exerted by the prosthetic treatment of teeth.

\section{ACKNOWLEDGEMENTS}

This work contains part of the doctoral thesis of Daniela Calderón Carrión.

1 Lumpkin EA, Caterina MJ. Mechanisms of sensory transduction in the skin. Nature 2007; 445(7130): 858-865.

2 Julius D, Basbaum Al. Molecular mechanisms of nociception. Nature 2001; 413(6852): 203-210.

3 Walker RG, Willingham AT, Zuker CS. A Drosophila mechanosensory transduction channel. Science 2000; 287(5461): 2229-2234.

4 Haeberle $\mathrm{H}$, Fujiwara M, Chuang J et al. Molecular profiling reveals synaptic release machinery in Merkel cells. Proc Natl Acad Sci U S A 2004; 101(40): 14503-14508.

5 Lee H, Caterina MJ. TRPV channels as thermosensory receptors in epithelial cells. Pflugers Arch 2005; 451(1): 160-167.

6 Maksimovic S, Nakatani M, Baba Y et al. Epidermal Merkel cells are mechanosensory cells that tune mammalian touch receptors. Nature 2014; 509(7502): 617-621.

7 Woo SH, Ranade S, Weyer AD et al. Piezo2 is required for Merkel-cell mechanotransduction. Nature 2014; 509(7502): 622-626.

8 Hsieh ST, Lin WM. Modulation of keratinocyte proliferation by skin innervation. J Invest Dermatol 1999; 113(4): 579-586.

9 Liu JY, Hu JH, Zhu QG et al. Substance P receptor expression in human skin keratinocytes and fibroblasts. Br J Dermatol 2006; 155(4): 657-662.

10 Roggenkamp D, Köpnick S, Stäb F et al. Epidermal nerve fibers modulate keratinocyte growth via neuropeptide signaling in an innervated skin model. J Invest Dermatol 2013; 133(6): 1620-1628.

11 Hosoi J, Murphy GF, Egan CL et al. Regulation of Langerhans cell function by nerves containing calcitonin gene-related peptide. Nature 1993; 363(6425): 159-163.
12 Asahina A, Hosoi J, Grabbe S et al. Modulation of Langerhans cell function by epidermal nerves. J Allergy Clin Immunol 1995; 96(6 Pt 2): 1178-1182.

13 Kingsmill VJ, Berkovitz BK, Barrett AW. An immunohistochemical analysis of human Merkel cell density in gingival epithelium from dentate and edentulous subjects. Arch Oral Biol 2005; 50(10): 883-887.

14 Moll I, Kuhn C, Moll R. Cytokeratin 20 is a general marker of cutaneous Merkel cells while certain neuronal proteins are absent. J Invest Dermatol 1995; 104(6): 910-915.

15 Barrett AW, Cort EM, Patel P et al. An immunohistological study of cytokeratin 20 in human and mammalian oral epithelium. Arch Oral Biol 2000; 45(10): 879-887.

16 Korkmaz $\mathrm{Y}$, Lang $\mathrm{H}$, Beikler $\mathrm{T}$ et al. Irreversible inflammation is associated with decreased levels of the $\alpha 1-, \beta 1$-, and $\alpha 2$-subunits of SGC in human odontoblasts. $J$ Dent Res 2011; 90(4): 517-522.

17 Korkmaz Y, Bloch W, Klinz FJ et al. The constitutive activation of extracellular signalregulated kinase 1 and 2 in periodontal ligament nerve fibers. J Periodontol 2009; 80(5): 850-859.

18 Halata Z, Grim M, Bauman KI. Friedrich Sigmund Merkel and his "Merkel cell", morphology, development, and physiology: review and new results. Anat Rec A Discov Mol Cell Evol Biol 2003; 271(1): 225-239.

19 Suzuki Y, Matsuzaka K, Ishizaki K et al. Characterization of the peri-implant epithelium in hamster palatine mucosa: behavior of Merkel cells and nerve endings. Biomed Res 2005; 26(6): 257-269.

20 Hartschuh W, Weihe E, Egner U. Electron microscopic immunogold cytochemistry reveals chromogranin A confined to secretory granules of porcine Merkel cells. Neurosci Lett 1990; 116(3): 245-249.

21 Mihara M, Hashimoto K, Ueda K et al. The specialized junctions between Merkel cell and neurite: an electron microscopic study. J Invest Dermatol 1979; 73(5): 325-334.

22 Ikeda I, Yamashita Y, Ono T et al. Selective phototoxic destruction of rat Merkel cells abolishes responses of slowly adapting type I mechanoreceptor units. J Physio/ 1994; 479(Pt 2): 247-256.

23 Kinkelin I, Stucky CL, Koltzenburg M. Postnatal loss of Merkel cells, but not of slowly adapting mechanoreceptors in mice lacking the neurotrophin receptor p75. Eur J Neurosci 1999; 11(11): 3963-3969.

This work is licensed under a Creative Commons Attribution-

NonCommercial-NoDerivs 4.0 Unported License. The images or other third party material in this article are included in the article's Creative Commons license, unless indicated otherwise in the credit line; if the material is not included under the Creative Commons license, users will need to obtain permission from the license holder to reproduce the material. To view a copy of this license, visit http://creativecommons.org/licenses/ by-nc-nd/4.0/ 